\title{
Downregulation of IncRNA H19 inhibits migration and invasion of human osteosarcoma through the NF-kB pathway
}

\author{
JUN ZHAO ${ }^{1-3}$ and SHI-TANG MA ${ }^{4}$ \\ ${ }^{1}$ Department of Bone and Soft Tissue Tumor, National Clinical Research Center for Cancer; \\ ${ }^{2}$ Key Laboratory of Cancer Prevention and Therapy; ${ }^{3}$ Tianjin's Clinical Research Center of Cancer, \\ Tianjin Medical University Cancer Institute and Hospital, Hexi, Tianjin 300060; ${ }^{4}$ Food and Drug College, \\ Anhui Science and Technology University, Fengyang, Anhui 233100, P.R. China
}

Received September 27, 2017; Accepted February 12, 2018

DOI: $10.3892 / \mathrm{mmr} .2018 .8746$

\begin{abstract}
The present study aimed to investigate the role of long non-coding RNA (lncRNA) H19 in the development of osteosarcoma and to determine the underlying mechanism involved. A total of 40 patients with osteosarcoma were selected and the expression level of H19 in tumor tissue and adjacent healthy tissue was detected by reverse transcription-quantitative polymerase chain reaction. Survival curves were plotted using the Kaplan-Meier method to investigate the prognostic value of $\mathrm{H} 19$ expression level for patients with osteosarcoma. H19 knockdown osteosarcoma cell lines were constructed using small interfering (si)RNA transfection. Cell migration and invasion abilities were measured by Transwell migration and invasion assays, respectively. Western blot analysis was performed to detect the expression levels of phosphatidylinositol 3-kinase (PI3K), phospho (p)-PI3K, RAC-alpha serine/threonine-protein kinase (AKT), p-AKT and NF- $\kappa$ B inhibitor $\alpha(\mathrm{I} \mathrm{B} \alpha)$ in osteosarcoma cells transfected with H19 siRNA. Expression level of H19 was significantly elevated in tumor tissue compared with adjacent healthy tissue. Expression level of $\mathrm{H} 19$ was positively associated with distant metastasis of osteosarcoma $(\mathrm{P}<0.01)$, but not with gender and age. Overall survival of patients with osteosarcoma with high H19 level was significantly shorter compared with patients with low H19 expression $(\mathrm{P}<0.05)$. H19 knockdown significantly reduced migration and invasion ability of osteosarcoma cells. Significantly decreased levels of $\mathrm{p}$-PI3K and p-AKT, and elevated level of IкB $\alpha$ were observed in H19 knockdown osteosarcoma cells compared with control osteosarcoma cells, while no significant differences in levels
\end{abstract}

Correspondence to: Dr Shi-Tang Ma, Food and Drug College, Anhui Science and Technology University, 9 Donghua Road, Fengyang, Anhui 233100, P.R. China

E-mail: shitangma123@gmail.com

Key words: osteosarcoma, long noncoding RNA H19, migration, invasion, phosphatidylinositol 3-kinase/phosphorylated-AKT pathway, nuclear factor- $\kappa \mathrm{B}$ pathway of PI3K and AKT were observed. Therefore, the present study demonstrated that knockdown of IncRNA H19 can inhibit migration and invasion of human osteosarcoma cells by inhibiting the nuclear factor- $\kappa \mathrm{B}$ pathway.

\section{Introduction}

Although osteosarcoma is a relatively rare type of cancer, it is considered to be one of the major causes of cancer-associated mortality among children and young adults (1). In the USA, $\sim 1,000$ new cases of osteosarcoma are reported each year (2). Young patients are typically diagnosed with primary conventional osteosarcoma, while secondary osteosarcoma is more common among elderly patients (2). Osteosarcoma primarily affects the long bones, but other bones in the body can also be affected (3). Similar to other tumors, development of osteosarcoma is a complex process with various signaling pathways involved (4). Therefore, in-depth analyses of signal transduction involved regulation in tumorigenesis may aid in elucidation of molecular mechanisms underlying the onset, development and progression of osteosarcoma.

The role of noncoding RNAs in the development of various pathophysiological conditions has been well characterized (5). Long non-coding RNAs (lncRNA) are a type of noncoding RNAs of $>200$ nucleotides in length (6). lncRNAs have roles in the onset and development of different human cancer types, including breast cancer, non-small-cell lung cancer and colorectal cancer (7-10). Expression of lncRNA H19 has been reported to be positively associated with progression of various malignant human tumors $(11,12)$. It has been reported that H19 is frequently overexpressed during the development of osteosarcoma (13) and that $\mathrm{H} 19$ can promote migration and invasion of osteosarcoma cells (14). However, the molecular mechanism underlying the function of H19 in osteosarcoma remains to be elucidated.

In the present study, expression of H19 in osteosarcoma tumor tissue and adjacent healthy tissue was detected. Effects of H19 on migration and invasion of multiple osteosarcoma cells lines were determined. Association between H19 and the NF- $\mathrm{KB}$ signaling pathway was investigated. In addition, prognostic value of expression of $\mathrm{H} 19$ for patients with osteosarcoma was also evaluated. 


\section{Materials and methods}

Patients. A total of 40 patients with osteosarcoma were selected in Tianjin Medical University Cancer Institute and Hospital from January 2010 to January 2013. All patients were diagnosed based on pathological and imaging examinations. Inclusion criteria were: i) Patient pathologically diagnosed as osteosarcoma and ii) patients willing to cooperate with researchers. Exclusion criteria were: i) Patient with other malignancies; ii) patients with metal disorders; iii) patients who had received treatment prior to admission. Surgical resection was performed for all patients. The patients included 18 males and 22 females (age range 8-66 years; mean age of $31 \pm 8.1$ years). Distant metastasis was observed in 22 patients. During surgical operations, cancer tissues and normal tissues within the region $0.5 \mathrm{~cm}$ around tumor were collected for subsequent experiments. The present study was approved by the Ethics Committee of Tianjin Medical University Cancer Institute and Hospital (Tianjin, China), and all patients signed informed consent. A follow-up study was performed for 48 months to monitor the survival.

Cell lines and cell culture. Human MG-63, U2OS and SAOS-2 osteosarcoma cell lines, and hFOB normal bone cell line, were purchased from American Type Culture Collection (Manassas, VA, USA). MG-63 and SAOS-2 cells were cultured with Eagle's minimum Essential medium (cat. no. 30-2003; American Type Culture Collection) containing 10\% heat-inactivated fetal bovine serum (Thermo Fisher Scientific, Inc., Waltham, MA, USA). U2OS and SAOS-2 cells were cultured with McCoy's 5a modified medium (cat. no. 30-2007; American Type Culture Collection) containing $10 \%$ fetal bovine serum (Thermo Fisher Scientific, Inc.). All cells were cultured at $37^{\circ} \mathrm{C}$ and $5 \% \mathrm{CO}_{2}$. Cells were harvested during logarithmic growth phase for subsequent experiments.

Reverse transcription-quantitative polymerase chain reaction $(R T-q P C R)$. TRIzol reagent (Invitrogen; Thermo Fisher Scientific, Inc.) was used to extract total RNA from tissues and cells. Concentration and quality of RNA samples were determined using a NanoDrop ${ }^{\text {TM }} 2000$ Spectrophotometer (Thermo Fisher Scientific, Inc.). Only RNA samples with a ratio of A260/A280 between 1.8 and 2.0 were used for reverse transcription to synthesize cDNA using SuperScript III Reverse Transcriptase (Thermo Fisher Scientific, Inc.). Reaction conditions for reverse transcription were: $25^{\circ} \mathrm{C}$ for $5 \mathrm{~min}, 55^{\circ} \mathrm{C}$ for $20 \mathrm{~min}$ and $80^{\circ} \mathrm{C}$ for $10 \mathrm{~min}$. SYBR ${ }^{\circledR}$-Green Real-Time PCR Master Mix (Thermo Fisher Scientific, Inc.) was used for qPCR. The following primers were used in PCR reactions: 5'-TGAGCTCTCAGGAGGGAGGATGG-3' (forward) and 5'-TTGTCACGTCCACCGGACCTG-3' (reverse) for H19; 5'-GACCTCTATGCCAACACAGT-3' (forward) and 5'-AGT ACTTGCGCTCAGGAGGA-3' (reverse) for $\beta$-actin. PCR reactions were performed using CFX96 Touch $^{\mathrm{TM}}$ Real-Time PCR Detection System (Bio-Rad Laboratories, Inc., Hercules, CA, USA). The following thermocycling conditions were used for the PCR: Initial denaturation at $95^{\circ} \mathrm{C}$ for $40 \mathrm{sec} ; 40$ cycles of $95^{\circ} \mathrm{C}$ for $15 \mathrm{sec}$ and $60^{\circ} \mathrm{C}$ for $45 \mathrm{sec}$. Relative expression was calculated using $2^{-\Delta \triangle \mathrm{Cq}}$ method (15). Relative expression level of $\mathrm{H} 19$ was normalized to endogenous control $\beta$-actin.
Establishment of H19-silenced cell lines. H19 small interfering RNA (siRNA; cat. no. 1299001; Thermo Fisher Scientific, Inc.) and Silencer ${ }^{\text {TM }}$ Select Negative Control No. 1 siRNA (cat. no. 4390843; Thermo Fisher Scientific, Inc.) were used. Cells were cultured overnight to reach $80-90 \%$ confluence prior to transfection. Transfection (50 nM siRNA) with Lipofectamine ${ }^{\circledR}$ 2000 reagent (Invitrogen; Thermo Fisher Scientific, Inc.) was performed according to the manufacturer's protocol. Cells were cultured for another $6 \mathrm{~h}$ before subsequent experiments.

Cell migration and invasion assay. Transwell cell migration assay (BD Biosciences, Franklin Lakes, NJ, USA) was performed to measure cell migration ability. Briefly, $5 \times 10^{4}$ cells in $100 \mu \mathrm{l}$ serum-free Dulbecco's modified Eagle medium (Thermo Fisher Scientific) were transferred to the upper chamber, while the lower chamber was filled with RPMI-1640 medium (Thermo Fisher Scientific, Inc.) containing 20\% fetal calf serum (Sigma-Aldrich; Merck KGaA, Darmstadt, Germany). Membranes were collected $24 \mathrm{~h}$ later and stained with $0.5 \%$ crystal violet at room temperature (Sigma-Aldrich; Merck KGaA) for $20 \mathrm{~min}$. Stained cells were counted under a light microscope (Olympus Corporation, Tokyo, Japan). Invasion assay was performed using the same method with the upper chamber was pre-coated with Matrigel (EMD Millipore, Billerica, MA, USA).

Western blot analysis. Total protein extraction was performed using RIPA buffer (Thermo Fisher Scientific, Inc.), and protein concentration was measured using THE bicinchoninic acid method. Subsequently, $30 \mu \mathrm{g}$ of protein was subjected to $10 \%$ SDS-PAGE gel electrophoresis, and transferred to polyvinylidene fluoride membrane at $20 \mathrm{~V}$ for $1 \mathrm{~h}$. Following blocking with 5\% skimmed milk at room temperature for $1 \mathrm{~h}$, membranes were washed with TBST $(0.1 \%$ Tween 20$) 3$ times for $15 \mathrm{~min}$ each time. Membranes were subsequently incubated with primary antibodies, including rabbit anti-p-PI3K (1:2,000; cat. no. ab182651), anti-PI3K (1:2,000; cat. no. ab5451; Abcam), anti-p-AKT (1:2,000; cat. no. ab18206; Abcam), anti-AKT (1:2,000; cat. no. ab126811; Abcam), anti-NF-кB inhibitor $\alpha(\mathrm{I} \kappa \mathrm{B} \alpha$; 1:1,000; cat. no. ab76429; Abcam) and anti-GAPDH (1:1,000; ab8245; all Abcam, Cambridge, UK) overnight at $4^{\circ} \mathrm{C}$. Subsequently, membranes were washed three times with TBST, 15 min each time. Membranes were incubated with anti-rabbit immunoglobulin G-horseradish peroxidase conjugated secondary antibody (1:1,000; cat. no. MBS435036; MyBioSource, Inc., San Diego, CA, USA) at room temperature for $2 \mathrm{~h}$. Following washing twice with TBST, 15 min each time, Enhanced Chemiluminescence detection reagent (Sigma-Aldrich; Merck KGaA) was used to detect the signals. Images were analyzed using Image J software version 1.8.0 (National Institutes of Health, Bethesda, MD, USA) to calculate relative expression level of each protein relative to endogenous control $\beta$-actin.

Statistical analysis. SPSS software (version 19.0; IBM Corp., Armonk, NY, USA) was used for all statistical analysis. Normal distribution data are presented as the mean \pm standard deviation. Comparisons between two groups were performed using t test and comparisons among multiple groups were performed using one-way analysis of variance and least 
Table I. Correlation between the expression level of H19 and clinicopathological features.

\begin{tabular}{lccccc}
\hline & & & & \multicolumn{2}{c}{ H19 expression } \\
\cline { 5 - 6 } Clinicopathological feature & Group & Total no. & High & Low & P-value \\
\hline Sex & Male & 18 & 8 & 10 & 0.79 \\
\multirow{2}{*}{ Age (years) } & Female & 22 & 12 & 10 & 0.72 \\
& $>25$ & 13 & 6 & 7 & $<0.01$ \\
Distant metastasis & $\leq 30$ & 27 & 11 & 16 & 5 \\
& Yes & 22 & 4 & 14 & \\
\hline
\end{tabular}

Table II. Multivariate regression analysis of factors associated with mortality of patients with osteosarcoma.

\begin{tabular}{lclc}
\hline Clinicopathological feature & Risk ratio & $95 \%$ CI & P-value \\
\hline Gender (male) & 1.02 & $0.61-5.52$ & 0.33 \\
Age (>40) & 3.83 & $2.12-7.73$ & 0.04 \\
Distant metastasis & 6.92 & $2.85-12.82$ & 0.01 \\
High H19 expression level & 6.53 & $2.27-9.58$ & 0.01 \\
\hline
\end{tabular}

CI, confidence interval.

significant difference test. Data that were not normally distributed were analyzed using non-parametric Mann-Whitney U test. In multivariate logistic regression analysis, mortality was set as dependent variable, and gender (being a male), age (>40), distant metastasis and high H19 expression level were independent variables. Survival curves were plotted using Kaplan-Meier method and compared using log-rank test. With the median expression level of H19 in cancer tissues as the cutoff score, 40 patients with osteosarcoma were divided into high expression level group $(n=20)$ and low expression level group $(n=20)$, and the association between the expression level of H19 and clinicopathological features was analyzed by $\chi^{2}$ test. $\mathrm{P}<0.05$ was considered to indicate a statistically significant difference.

\section{Results}

Comparison of expression levels of H19 in cancer tissues and adjacent healthy tissues. Expression levels of H19 in cancer tissues and adjacent healthy tissues of 40 patients with osteosarcoma were detected using RT-qPCR. Expression levels of H19 were significantly increased in cancer tissues of all 40 patients compared with the respective adjacent healthy tissues, indicating the potential role of H19 in development of osteosarcoma (Fig. 1).

Association between expression of H19 and clinicopathological features, and the prognostic values. Univariate analysis demonstrated that the expression level of H19 was positively associated with distant metastasis $(\mathrm{P}<0.01)$, not with gender and age (Table I). Multivariate regression analysis

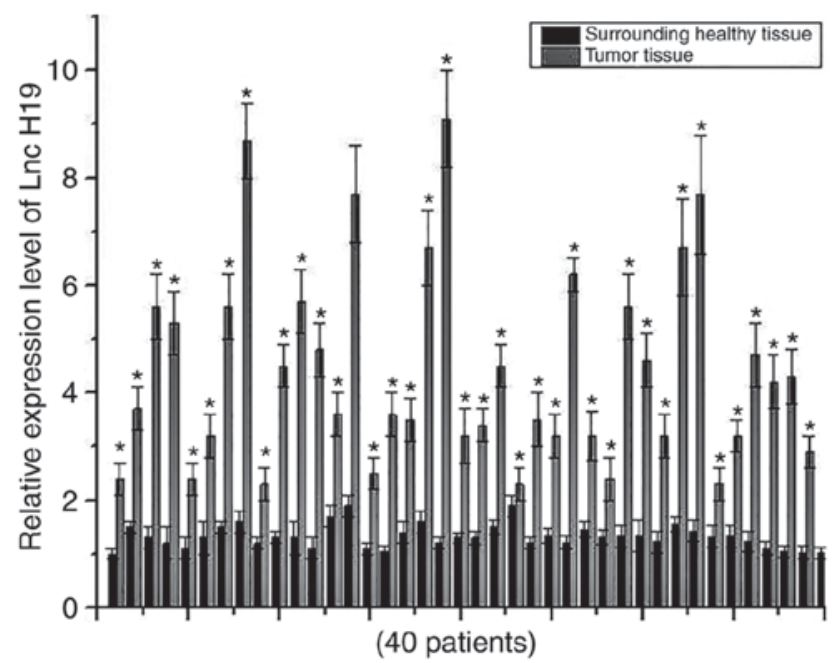

Figure 1. Expression of H19 in tumor tissues and adjacent healthy tissues. ${ }^{*} \mathrm{P}<0.05$ vs. the respective adjacent healthy tissue. lnc, long noncoding RNA.

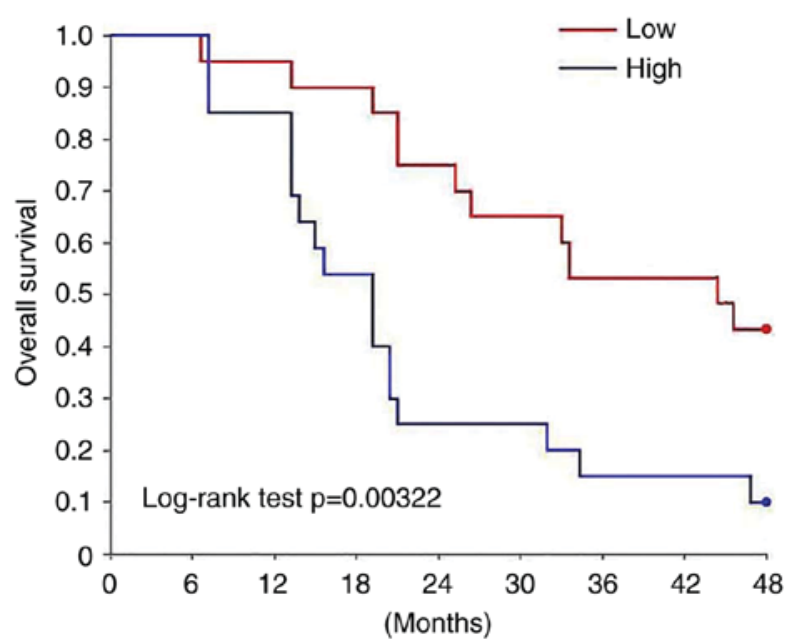

Figure 2. Overall survival curves plotted using Kaplan-Meier method for patients with high and low expression level of H19.

demonstrated that aging, distant metastasis and high expression level of $\mathrm{H} 19$ were associated with mortality of patients with osteosarcoma $(\mathrm{P}<0.05$; Table II). Survival curves were plotted using the Kaplan-Meier method to evaluate the prognostic value of H19 for osteosarcoma. Survival curves were 
A
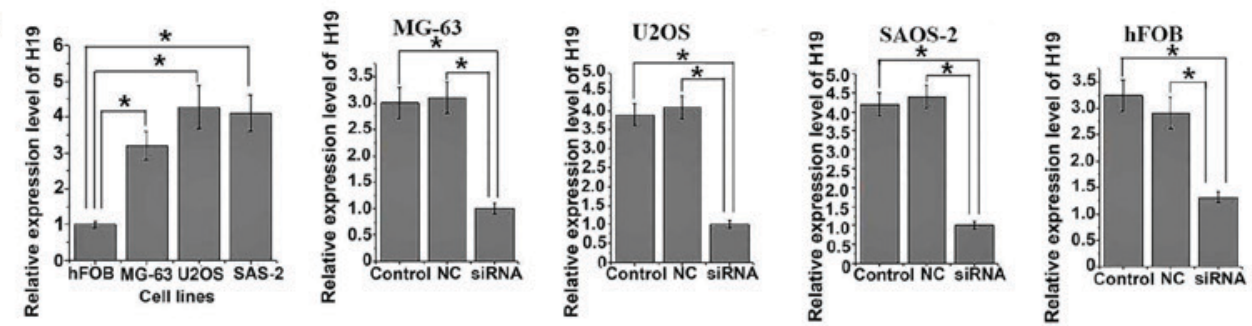

B

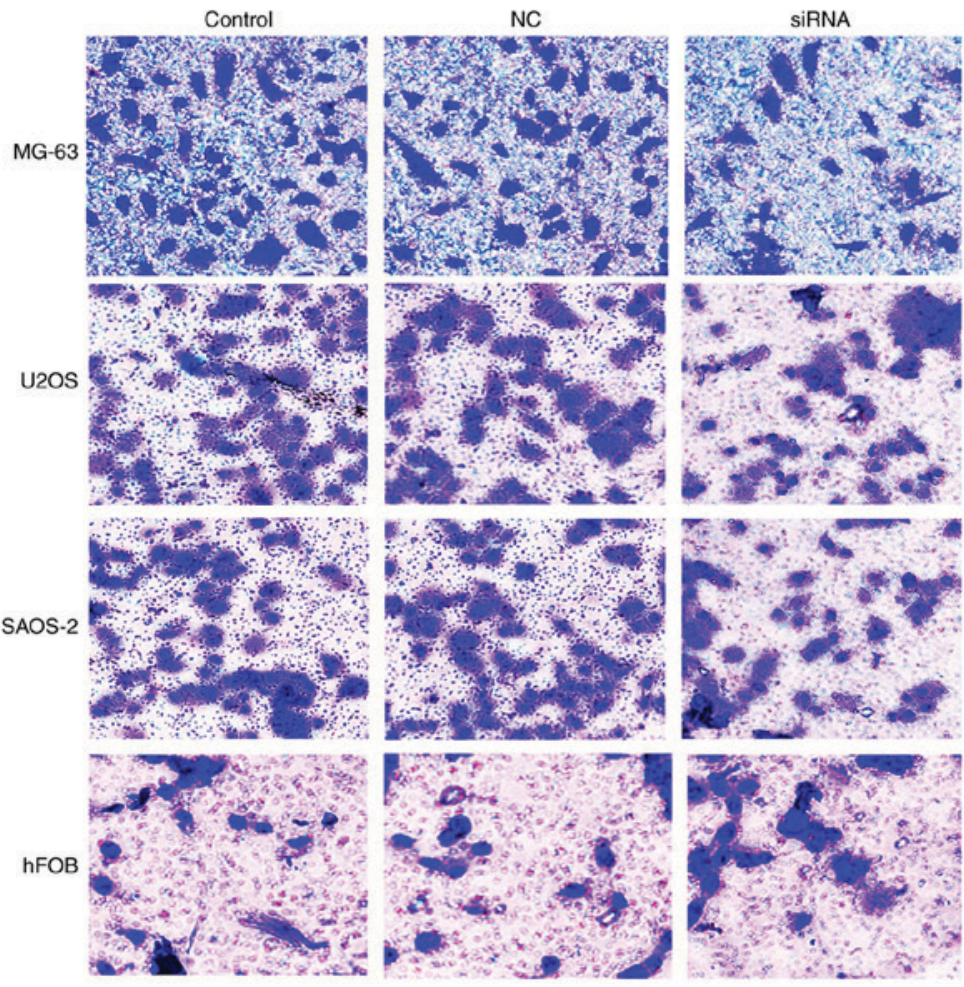

C

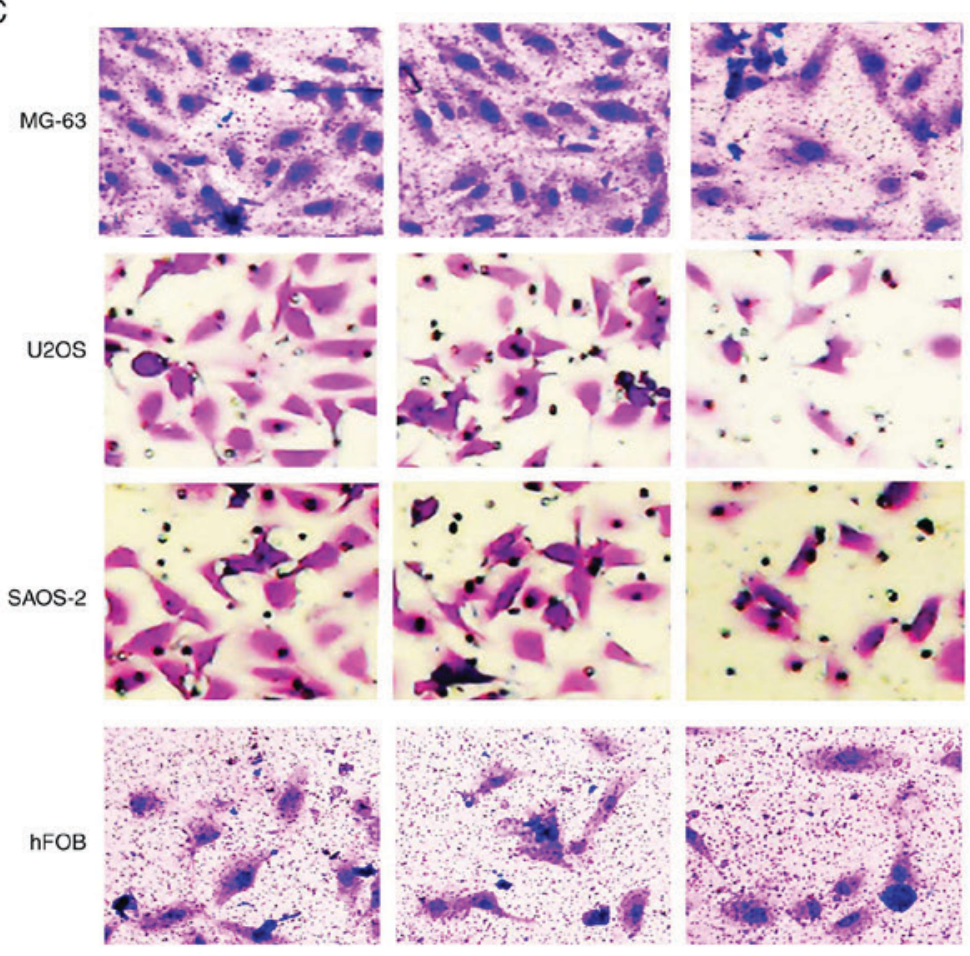

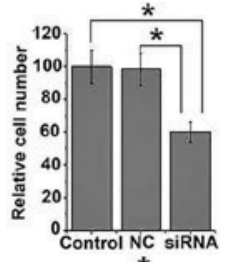
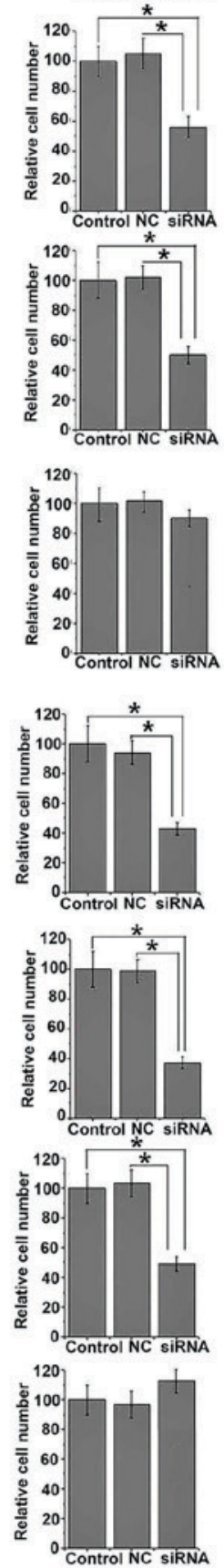

Figure 3. Effects of H19 knockdown on osteosarcoma cell migration and invasion. (A) Expression of H19 in different cell lines following different treatments. (B) Effects of H19 knockdown on osteosarcoma cell migration (magnification, x20). (C) Effects of H19 knockdown on osteosarcoma cell invasion (magnification, $\mathrm{x} 20$ ). Relative cell number was normalized to the control group, which was set as 100 . Scale bar $=20 \mu \mathrm{m}$. "P<0.05. NC, negative control; siRNA, small interfering RNA. 

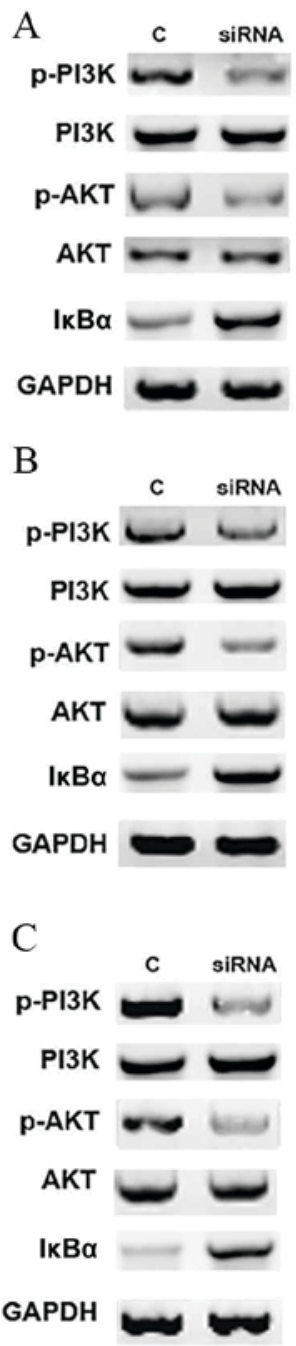
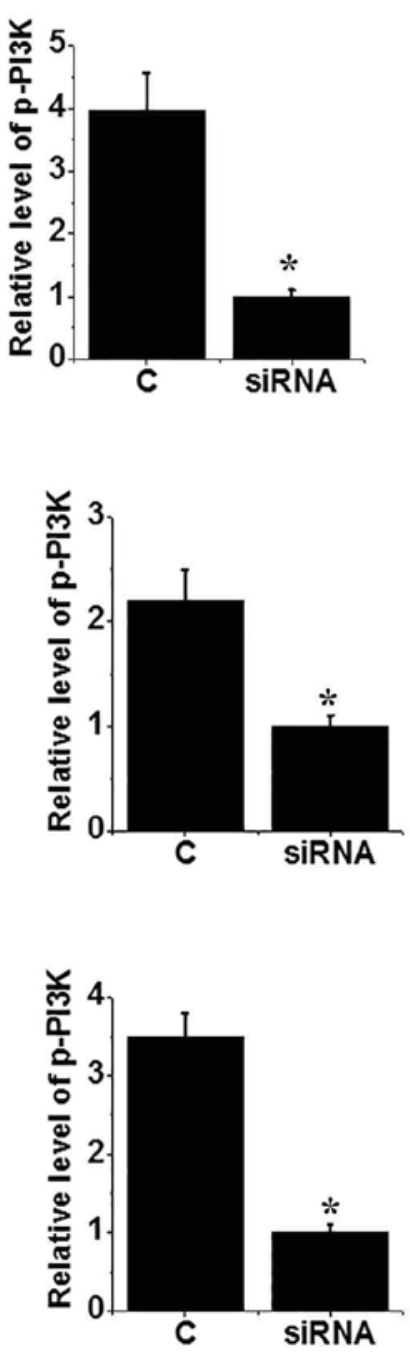
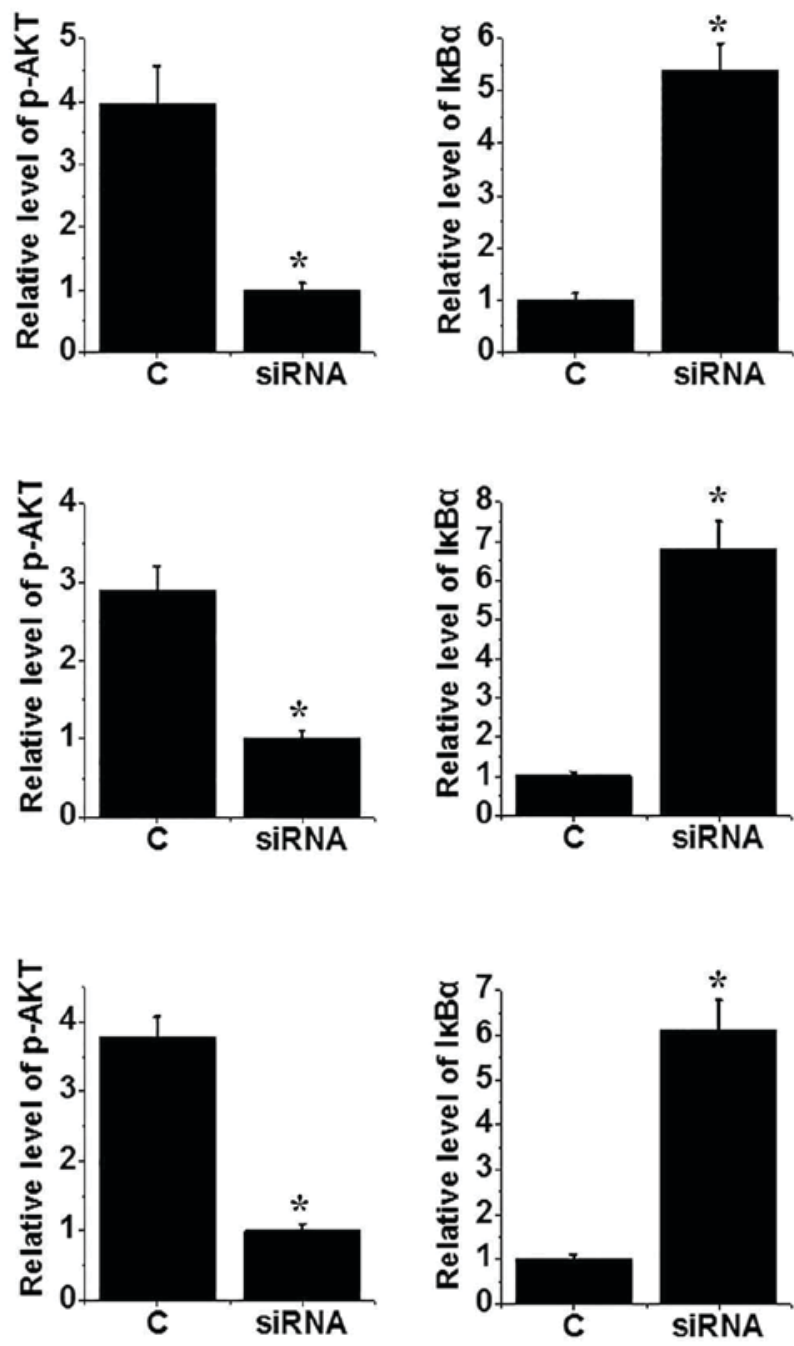

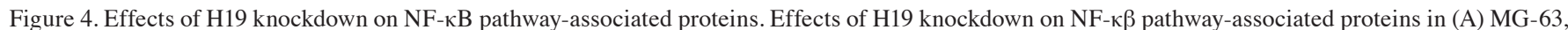

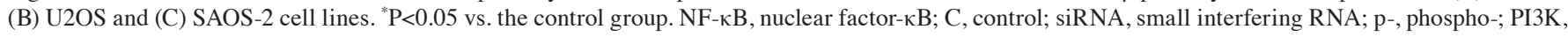
phosphatidylinositol 3-kinase; AKT, RAC-alpha serine/threonine-protein kinase; IкB $\alpha, \mathrm{NF}-\kappa \mathrm{B}$ inhibitor $\alpha$.

compared using log-rank test. The overall survival rate of patients with high expression level of H19 was significantly lower compared with patients with low expression level of H19 (P<0.05; Fig. 2).

Effects of H19 knockdown on osteosarcoma cell migration and invasion. Expression level of H19 was significantly increased in human osteosarcoma cell lines MG-63, U2OS and SAOS-2 compared with normal bone cell line hFOB (all $\mathrm{P}<0.05$; Fig. 3A). Following siRNA transfection, expression level of H19 was significantly reduced in all three osteosarcoma cell lines and in the normal hFOB cell line compared with the controls (all $\mathrm{P}<0.05$ ). Following siRNA H19 transfection, migration ability was significantly reduced in all osteosarcoma cell lines compared with control cells (all $\mathrm{P}<0.05)$. Similar results were observed in the invasion assay, where invasion ability of all osteosarcoma cell lines was significantly reduced following siRNA transfection (all $\mathrm{P}<0.05$ ). However, no significant differences in migration and invasion abilities were identified in hFOB cells with and without H19 knockdown (Fig. 3B and C).
Effects of H19 knockdown on the NF- $\mathrm{B} B$ signaling pathway. The PI3K/AKT signaling pathway can activate the NF- $\kappa \mathrm{B}$ pathway by regulating degradation of IкB $\alpha$. No significant differences in levels of PI3K and AKT were identified between the control and H19 knockdown groups of MG-64 (Fig. 4A), U2OS (Fig. 4B) and SAOS-2 (Fig. 4C) cells. Levels of p-PI3K and p-AKT were decreased significantly in cells transfected with siRNA compared with control cells $(\mathrm{P}<0.05)$, suggesting inactivation of the PI3K/AKT signaling pathway following H19 knockdown. In addition, the level of IкB $\alpha$ protein increased significantly in cells transfected with siRNA compared with control cells (all $\mathrm{P}<0.05$ ). The above data suggest that $\mathrm{H} 19$ knockdown can inactivate NF-kB pathway, potentially through interaction with the PI3K/AKT signaling pathway.

\section{Discussion}

The role of lncRNA in the development of osteosarcoma has been demonstrated by numerous studies $(14,16)$. Sun et al (14) reported that hepatocellular carcinoma up-regulated long 
non-coding RNA (HULC) was upregulated in osteosarcoma tissues compared with adjacent healthy tissues, and increased expression level of HULC was associated with a shorter overall survival of patients with osteosarcoma. By contrast, downregulation of HULC significantly reduced proliferation, migration and invasion of in vitro cultured osteosarcoma cells (14). IncRNA taurine up-regulated 1 (TUG1) was also demonstrated to inhibit apoptosis and promote proliferation of osteosarcoma cells, and TUG1 may be a novel target for treatment of osteosarcoma (16). H19 is an oncogenic lncRNA and was demonstrated to be involved in the progression of various types of human cancer $(11,12)$. A previous study reported that activation of the Hedgehog signaling pathway upregulated the expression of H19, which in turn promoted tumorigenesis (13). Consistent with previous studies, in the present study, the expression level of H19 in 40 osteosarcoma patients was increased markedly in tumor tissue compared with adjacent healthy tissue. The results suggest that H19 may be involved in development of osteosarcoma. In addition, the expression level of H19 was positively associated with the occurrence of distant metastasis of osteosarcoma, but not with gender or age, and the overall survival of patients with osteosarcoma exhibiting high H19 expression significantly shorter compared with patients with low expression H19. Thus, the results of the present study suggest that increased expression of H19 may have a prognostic role for patients with distant metastasis of osteosarcoma.

lncRNA H19 serves a role in the progression of different types of cancer primarily by promoting tumor metastasis. Shi et al (17) reported that miR-675 derived from H19 can promote invasion of glioma cells. In another study, H19 promoted migration and invasion of human hepatocellular carcinoma cells by activating with the AKT/GSK-3 $\beta / \mathrm{Cdc} 25 \mathrm{~A}$ signal transduction pathway (18). In the present study, H19 knockdown reduced migration and invasion abilities of three osteosarcoma cell lines. The results of the present study suggest that expression level of H19 is positively associated with invasion and migration abilities of osteosarcoma cells.

$\mathrm{NF}-\kappa \mathrm{B}$ signaling has roles in numerous aspects of initiation and progression of tumorigenesis (19). Previous studies demonstrated that $\mathrm{H} 19$ can interact with the $\mathrm{NF}-\kappa \mathrm{B}$ pathway to perform its biological functions (20). It has also been demonstrated that the NF- $\mathrm{NB}$ pathway can be activated by the PI3K/AKT signaling pathway via the regulation of degradation of $\mathrm{I} \kappa \mathrm{B} \alpha(21,22)$. In the present study, phosphorylation levels of PI3K and AKT decreased, and expression level of $\mathrm{I} \kappa \mathrm{B} \alpha$ increased following knockdown of H19, while no significant differences in expression levels of PI3K and AKT were identified between osteosarcoma cells with and without H19 knockdown. The above results suggest that knockdown of H19 can mediate inactivation of the NF- $\kappa \mathrm{B}$ pathway by inhibiting the activation PI3K/AKT pathway.

In conclusion, expression level of H19 increased in osteosarcoma tissues compared with adjacent healthy tissues. Expression level of H19 was positively associated with the occurrence of distant metastasis, and elevated expression level of H19 indicated poor prognosis of patients with osteosarcoma. Expression of $\mathrm{H} 19$ affected migration and invasion of osteosarcoma by activating the NF- $\mathrm{NB}$ pathway. Future studies should focus on identifying targets of H19 to further elucidate the underlying mechanism of $\mathrm{H} 19$ in osteosarcoma. The present study was limited by small sample size. Further studies with larger sample sizes are necessary to confirm the conclusions of the present study.

\section{Acknowledgements}

Not applicable.

\section{Funding}

The present study was supported by Major Science and Technology Projects of Anhui Province (grant no. 17030801014).

\section{Availability of data and materials}

All data generated or analyzed during this study are included in this published article.

\section{Authors' contributions}

MST designed the experiments. ZJ performed the experiments. MST and ZJ analysed the data. MST wrote the manuscript. All authors read and approved the final manuscript.

\section{Ethics approval and consent to participate}

The present study was approved by the Ethics Committee of Tianjin Medical University Cancer Institute and Hospital (Tianjin, China), and all patients signed informed consent.

\section{Consent for publication}

All participants signed informed consent.

\section{Competing interests}

The authors declare that they have no competing interests.

\section{References}

1. Botter SM, Neri D and Fuchs B: Recent advances in osteosarcoma. Curr Opin Pharmacol 16: 15-23, 2014.

2. Lin PP and Patel S (eds): Osteosarcoma. In: Bone Sarcoma. Springer, New York, NY, pp75-97, 2013.

3. Moore DD and Luu HH (eds): Osteosarcoma. In: Orthopaedic Oncology. Springer, New York, NY, pp65-92, 2014.

4. Akiyama T, Dass CR and Choong PF: Novel therapeutic strategy for osteosarcoma targeting osteoclast differentiation, bone-resorbing activity, and apoptosis pathway. Mol Cancer Ther 7: 3461-3469, 2008.

5. Esteller M: Non-coding RNAs in human disease. Nat Rev Genet 12: 861-874, 2011.

6. Perkel JM: Visiting 'noncodarnia'. Biotechniques 54: 301, 2013.

7. Ning S, Zhang J, Wang P,Zhi H, Wang J,Liu Y, Gao Y, Guo M, Yue M, Wang L and Li X: Lnc2Cancer: A manually curated database of experimentally supported lncRNAs associated with various human cancers. Nucleic Acids Res 44: D980-D985, 2016.

8. Augoff K, McCue B, Plow EF and Sossey-Alaoui K: miR-31 and its host gene lncRNA LOC554202 are regulated by promoter hypermethylation in triple-negative breast cancer. Mol Cancer 11: 5, 2012.

9. Yang Y, Li H, Hou S, Hu B, Liu J and Wang J: The noncoding RNA expression profile and the effect of lncRNA AK126698 on cisplatin resistance in non-small-cell lung cancer cell. PLoS One 8: e65309, 2013. 
10. Zheng HT, Shi DB, Wang YW, Li XX, Xu Y, Tripathi P, Gu WL, Cai GX and Cai SJ: High expression of lncRNA MALAT1 suggests a biomarker of poor prognosis in colorectal cancer. Int J Clin Exp Pathol 7: 3174-3181, 2014.

11. Yang F, Bi J, Xue X, Zheng L, Zhi K, Hua J and Fang G: Up-regulated long non-coding RNA H19 contributes to proliferation of gastric cancer cells. FEBS J 279: 3159-3165, 2012.

12. Luo M, Li Z, Wang W, Zeng Y, Liu Z and Qiu J: Long non-coding RNA H19 increases bladder cancer metastasis by associating with EZH2 and inhibiting E-cadherin expression. Cancer Lett 333: 213-221, 2013.

13. Chan LH, Wang W, Yeung W, Deng Y, Yuan P and Mak KK Hedgehog signaling induces osteosarcoma development through Yap1 and H19 overexpression. Oncogene 33: 4857-4866, 2014.

14. Sun XH, Yang LB, Geng XL, Wang R and Zhang ZC: Increased expression of lncRNA HULC indicates a poor prognosis and promotes cell metastasis in osteosarcoma. Int J Clin Exp Pathol 8: 2994-3000, 2015.

15. Livak KJ and Schmittgen TD: Analysis of relative gene expression data using real-time quantitative PCR and the 2(-Delta Delta C (T)) method. Methods 25: 402-408, 2001.

16. Zhang Q, Geng PL, Yin P, Wang XL, Jia JP and Yao J: Down-regulation of long non-coding RNA TUG1 inhibits osteosarcoma cell proliferation and promotes apoptosis. Asian Pac J Cancer Prev 14: 2311-2315, 2013.
17. Shi Y, Wang Y, Luan W, Wang P, Tao T, Zhang J, Qian J, Liu N and You Y: Long non-coding RNA H19 promotes glioma cell invasion by deriving miR-675. PLoS One 9: e86295, 2014.

18. Lv J, Ma L, Chen XL, Huang XH and Wang Q: Downregulation of LncRNAH19 and MiR-675 promotes migration and invasion of human hepatocellular carcinoma cells through AKT/GSK-3 $\beta / C d c 25 A$ signaling pathway. J Huazhong Univ Sci Technolog Med Sci 34: 363-369, 2014

19. Hoesel B and Schmid JA: The complexity of NF-kB signaling in inflammation and cancer. Mol Cancer 12: 86, 2013.

20. Pan JX: LncRNA H19 promotes atherosclerosis by regulating MAPK and NF-kB signaling pathway. Eur Rev Med Pharmacol Sci 21: 322-328, 2017.

21. Hyam SR, Lee IA, Gu W, Kim KA, Jeong JJ, Jang SE, Han MJ and Kim DH: Arctigenin ameliorates inflammation in vitro and in vivo by inhibiting the PI3K/AKT pathway and polarizing M1 macrophages to M2-like macrophages. Eur J Pharmacol 708: 21-29, 2013.

22. Han W, Xiong Y, Li Y, Fang W, Ma Y, Liu L, Li F and Zhu X: Anti-arthritic effects of clematichinenoside (AR-6) on PI3K/Akt signaling pathway and TNF- $\alpha$ associated with collagen-induced arthritis. Pharm Biol 51: 13-22, 2013. 